\title{
Original Article \\ Multicenter International Survey on the Clinical Practice of Ultra-Fast-Track Anesthesia with On-Table Extubation in Pediatric Congenital Cardiac Surgery
}

\author{
Mohammad Irfan Akhtar, MBBS, FCPS ${ }^{*,}{ }^{1}$, \\ Mona Momeni, $\mathrm{MD}, \mathrm{PhD}^{\dagger}$, Andrea Szekely, MD, PhD, $\mathrm{DEAA}^{\ddagger}$, \\ Mohammad Hamid, MBBS, DABA*, Mohamed R. El Tahan, MD ${ }^{\S}$, \\ Steffen Rex, MD, PhD ${ }^{\mathbb{T}, \|}$ \\ *Department of Anesthesiology, Aga Khan University Hospital, Karachi, Pakistan \\ ${ }^{\dagger}$ Department of Anesthesiology, Cliniques Universitaires Saint-Luc, Université catholique de Louvain, Brus- \\ sels, Belgium \\ ${ }^{\ddagger}$ Department of Anesthesiology and Intensive Care, Semmelweis University, Budapest, Hungary \\ ${ }^{\S}$ Department of Anesthesiology, King Fahd Hospital of Imam Abdulrahman Bin Faisal University, Dammam, \\ Saudi Arabia \\ "Department of Anesthesiology, University Hospitals Leuven, Leuven, Belgium \\ "Department of Cardiovascular Sciences, Katholieke Universiteit Leuven, Leuven, Belgium
}

\begin{abstract}
Objective: To describe global practices for on-table extubation (OTE) in pediatric cardiac anesthesia in European and non-European countries. Design: Multiple-choice, web-based survey with 34 questions addressing organizational data, existence of OTE programs, inclusion and exclusion criteria for OTE, and intraoperative and immediate postoperative management.

Setting: Online survey endorsed by the European Association of Cardiothoracic Anesthesiologists.

Participants: Anesthesiologists departments in European and non-European pediatric cardiac surgical centers.

Interventions: None.

Measurements and Main Results: The survey was sent to 144 pediatric cardiac surgical centers in 29 countries as a web-based questionnaire. Addressees were pediatric cardiac anesthesiologists who were members of European Association of Cardiothoracic Anesthesiologists or were known to the authors. The response rate was $63 \%$. Fifty percent of the respondents were practicing in university hospitals. The survey demonstrated that $76 \%$ of the respondents practiced OTE, with $50 \%$ of the pediatric cardiac anesthesiologists regularly performing OTE in different proportions, ranging from 1 to $51 \%$ of on-pump pediatric cardiac surgeries. Seventy-seven percent of respondents made their decision to perform OTE on an individual case-by-case basis. Seventy-eight percent of the congenital cardiac lesions deemed eligible for OTE fell into Risk Adjustment for Congenital Heart Surgery-1 categories 1 and 2. In patients for whom OTE was planned, anesthesia primarily was maintained using a combined inhalational and intravenous technique. The main reasons not to perform OTE were that it was deemed to provide no major advantage (45\%), to be dangerous (9\%), or to decrease operating room efficiency by increasing operating room turnover time $(36 \%)$.

Conclusion: The survey demonstrated that the majority of the approached pediatric cardiac anesthesiologists practice OTE regularly in pediatric cardiac surgery. Frequency of OTE and inclusion criteria vary widely. The observations made in this survey should prompt appropriately powered, randomized controlled clinical trials to examine the effect of OTE on various effectiveness and safety outcomes.
\end{abstract}

(C) 2018 Elsevier Inc. All rights reserved.

\footnotetext{
${ }^{1}$ Address reprint requeststo Mohammad Irfan Akhtar, MBBS, FCPS, Department of Anesthesiology, Aga Khan University Hospital, Stadium Road, PO Box 3500, Karachi, Pakistan.

E-mail address: mohammad.irfan@aku.edu (M.I. Akhtar).
} 
IN ADULTS, fast-track cardiac anesthesia has gained increasing popularity and has been shown to decrease the time to extubation and intensive care unit (ICU) length of stay. ${ }^{1}$ Even though they are adequately powered, prospective clinical trials with mortality as a primary end-point are not available. In a retrospective study, Ender et al observed a decrease in mortality when fast-track concepts, including implementation of direct admission to the postanesthetic care unit compared with standard perioperative management, were applied. ${ }^{2}$ The mortality benefit was attributed to the significantly shorter intubation and ventilation times after fast-track anesthesia and hence a lower risk for ventilator-associated infections and sepsis.

Recently, even ultra-fast track anesthesia [defined as on table extubation (OTE) in the operating room] has been advocated with, however, controversial effects on various outcomes. $^{3-5}$ Ultra-fast-track anesthesia in children with congenital heart disease (CHD) also recently has gained interest. ${ }^{6}$ Arguments in favor of early extubation in congenital pediatric cardiac surgery include the rapid re-establishment of normal physiologic conditions (eg, spontaneous ventilation facilitating pulmonary blood flow in patients with a Fontan circulation); early start of feeding; early interaction with parents; and a decreased incidence of ventilator-associated complications. ${ }^{7}$ In fact, in children, early extubation including OTE was demonstrated to be associated with low morbidity rates and a shorter length of stay in the ICU and hospital. ${ }^{8}$ The use of OTE has been encouraged, especially in patients undergoing repair of simple atrial and ventricular septal defects, although it also has been shown to be feasible in complex surgical cases. ${ }^{9}$ There is, however, reluctance among many pediatric cardiac anesthesiologists toward OTE in children with CHD, mainly because of concerns about possible extubation failure, probable hemodynamic instability resulting from insufficient hemostasis or bleeding, and the effect of OTE on turnover times within the OR that might affect efficiency in busy congenital centers. The principal goal of this online survey was to describe global ultra-fast-track pediatric cardiac anesthesia practices with OTE.

\section{Materials and Methods}

This cross-sectional survey was performed after approval from the Scientific Committee of the European Association of Cardiothoracic Anesthesiologists (EACTA).

\section{Survey Development}

The questionnaire was designed by members of the Pediatric Congenital Cardiac Subcommittee of the EACTA using the webbased platform SurveyMonkey (Palo Alto, CA). A preliminary survey was tested on a group of 5 pediatric cardiac anesthesiologists ( 3 EACTA and 2 non-EACTA members). Based on their feedback, several changes, including addition and deletion of some questions, alterations in question order, and corrections of language and text, were made before distribution.

\section{Survey Distribution}

An e-mail invitation that included a short description of the aim of the survey and contained a hyperlink to the online questionnaire was sent between January 15 and March 18, 2018, to 144 pediatric cardiac anesthesiologists working in pediatric cardiac centers in 29 countries in Europe (Germany, France, Italy, United Kingdom, Belgium, The Netherlands, Denmark, Sweden, Switzerland, Austria, Slovenia, Turkey, Greece); North America (Canada, United States); Africa (Egypt, South Africa); Asia (Pakistan, India, Singapore, Oman, Qatar, Saudi Arabia, United Arab Emirates, Lebanon, Bahrain, Hong Kong); New Zealand; and Australia. Addressees were pediatric cardiac anesthesiologists who were members of EACTA or known to the authors. Only 1 anesthesiologist per cardiac center was contacted. The survey was kept open for 2 months, during which time weekly e-mail reminders were sent to nonrespondents and participants who had given only partial responses.

\section{Questionnaire}

The web-based questionnaire comprised 34 questions and took an average time of 5 to 15 minutes to complete. The questionnaire included 3 sets of questions investigating the participant's organizational details, intraoperative OTE strategies, and postoperative management options for OTE pediatric cardiac surgical patients. The first set of questions was related to the participant's country of origin; organizational details, including the type of cardiac facility (university or private) with which the participants were affiliated; pediatric cardiac surgical volume; complexity of cardiac cases; existence of OTE programs; inclusion and exclusion criteria for OTE; and safety and feasibility. The second set of questions assessed anesthetic techniques for the maintenance of anesthesia; cardiopulmonary bypass (CPB) strategies (target temperature, use of modified ultrafiltration); and intraoperative prophylaxis for postoperative nausea and vomiting. The third set of questions included issues covering postoperative care (eg, unit to which patients are admitted, estimated length of stay, management of postoperative analgesia and respiratory support). Finally, participants were asked to provide reasons for withdrawing from an existing OTE program or not practicing OTE at all.

Because of the descriptive nature of the survey, no formal statistical analysis was performed, and results (absolute frequencies and percentages) were analyzed automatically with SurveyMonkey.

\section{Results}

The response rate from the participating countries is illustrated in Figure 1. One hundred centers from 29 countries responded to the survey ( 9 of which were only partial responses), resulting in an overall response rate of $63 \%$ (91 of 144). 


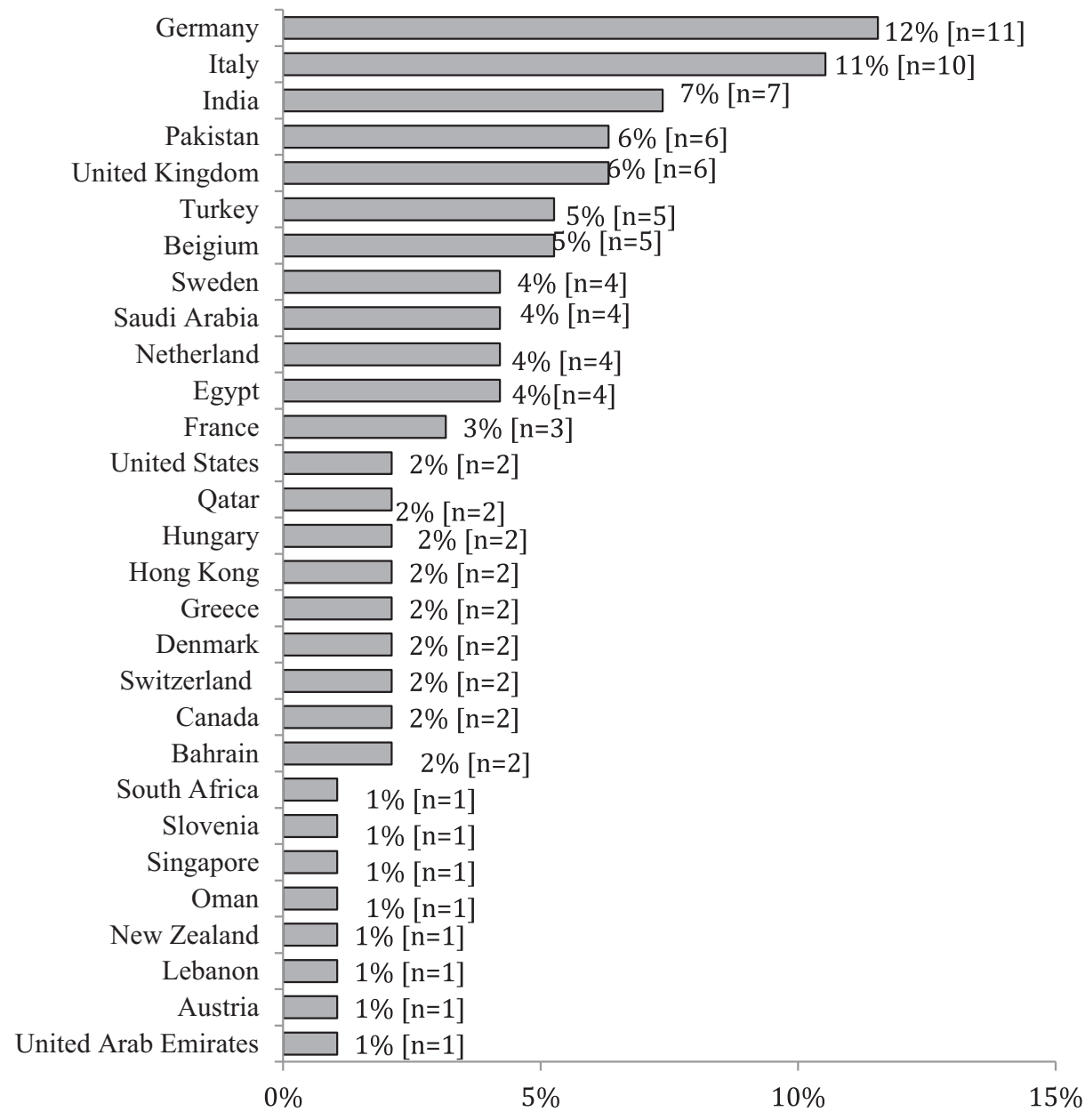

Fig. 1. Participating countries (29) and their response rate (percentage of total).

\section{Characteristics of Study Participants}

Fifty percent of the respondents were practicing in university hospitals, and $23 \%$ were affiliated with dedicated heart centers. Eighty-nine percent ( 81 of 91) of the respondents practiced on-pump surgery in $>50 \%$ of their pediatric cardiac surgeries. Two-thirds (59 of 91) of respondents reported that $>20$ patients younger than 16 years old undergo cardiac surgery at their centers each month. Complexity of surgeries was variable (Fig. 2).

\section{Experience with OTE}

Sixty-one percent ( 69 of 91) of the responding pediatric cardiac anesthesiologists were practicing OTE, and 24\% (21 of 91) had never practiced OTE. The practice of OTE was infrequent according to $48 \%$ (33 of 69) of the OTE practitioners, whereas $51 \%$ (35 of 69) were regularly practicing OTE in different proportions ranging from $1 \%$ to $10 \%$ to $>51 \%$ of their off-pump and on-pump pediatric cardiac cases (Fig. 3). Most of the OTE practicing pediatric anesthesiologists had started performing OTE more than 5 years previously (54\% [37 of 69]).

\section{OTE: Rationale, Indications, and Contraindications}

The majority of OTE practitioners (68\% [47 of 69]) performed OTE with the aim of reducing ventilation times and increasing ICU turnover, whereas 61\% (42 of 69) were practiced OTE primarily for clinical considerations (avoidance of positive pressure ventilation in patients with pulmonary hypertension or Fontan circulation).

Most respondents performed OTE in cases with lower complexity as assessed using the Risk Adjustment for Congenital Heart Surgery-1 (RACHS-1) categories, with category 1 being selected for OTE by $46 \%$ (32 of 69) and category 2 by $32 \%$ (22 of 69) of the responding anesthesiologists, respectively. Fifteen percent (10 of 69) of the centers were practicing OTE in RACHS- 1 categories 1 to 3, whereas $6 \%$ (4 of 69) of the centers performed OTE in all RACHS-1 categories (Fig. 4).

Primary exclusion criteria for OTE included excessive bleeding, lower-than-expected oxygen saturations for the type of surgical correction or palliation, long CPB times (>120 minutes), and high inotropic or vasopressor support. Age was not an exclusion criterion for 55\% (38 of 69) of the OTE practitioners; however, 20\% (14 of 69) of the respondents considered age $<6$ months to be an exclusion criterion. Cardiology 


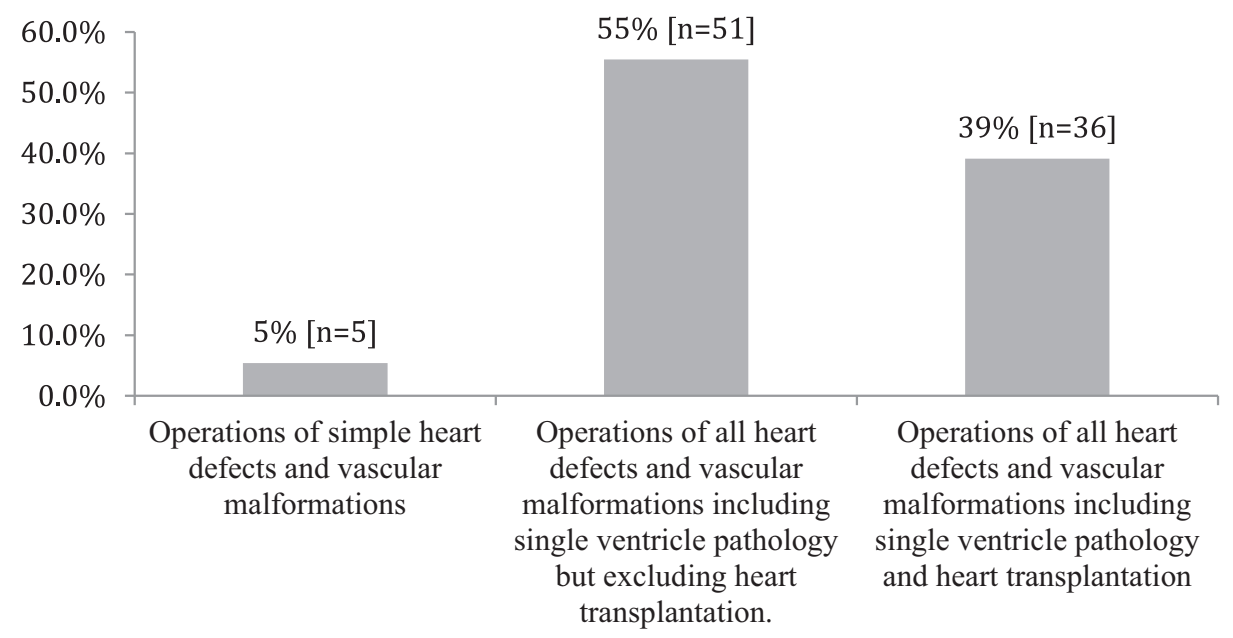

Fig. 2. Complexity of cardiac cases performed in the responding centers.

or pediatric intensive care unit (PICU) preferences and the presence of an open sternum were other exclusion criteria noted by $20 \%$ (14 of 69) of the OTE practitioners.

\section{Decision Making}

The decision regarding whether to perform OTE was made on an individual case-by-case basis by $77 \%$ (53 of 69) of the OTE practitioners. Formal criteria were followed by $7 \%$ ( 5 of 69 ) of the respondents, whereas $12 \%$ ( 8 of 69 ) of the respondents decided to include or exclude a patient into the OTE program based on informal criteria (eg, clinical experience, "gut feeling").

In $49 \%$ (34 of 69) of the pediatric cardiac centers practicing OTE, the decision to perform OTE was made by the pediatric cardiac anesthesiologist, whereas $48 \%$ (33 of 69) of the respondents relied on team decision.

\section{Intraoperative Management}

There was a wide variety in the intraoperative maintenance of anesthesia, including exclusive use of volatile anesthetics (20\% [14/69]), total intravenous anesthesia (10\% [7/69]), or a combination of both (70\% [48/69]). The most frequently used intravenous anesthetic agent for maintenance was propofol (66\% [36 of 55] of the responses), whereas 31\% (17 of 55) of pediatric cardiac anesthesiologists practicing OTE were using

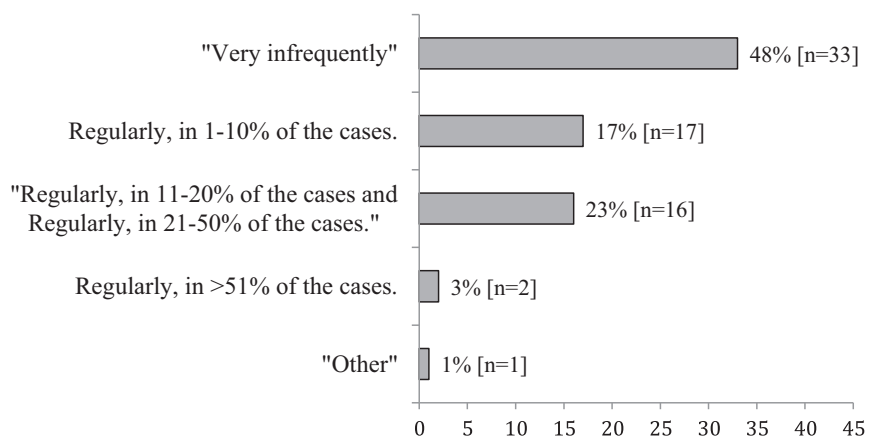

Fig. 3. Frequency with which on-table extubation is performed in various pediatric cardiac centers. benzodiazepines and 31\% (16 of 53) dexmedetomidine. Fiftyone percent ( 35 of 69 ) of the respondents were not administering prophylactic antiemetics, whereas $49 \%$ (34 of 69) did so as part of routine care.

Perioperative analgesia was achieved by administration of low-dose boluses of fentanyl (total dose $<20 \mu / \mathrm{kg}$ ) or an equivalent opioid, according to the majority of the OTE practitioners (Table 1). Fifty-seven percent (39 of 69) of the pediatric cardiac centers had never used regional anesthesia for intraoperative and postoperative analgesia. In the centers using regional anesthesia, the most frequently applied technique was local infiltration anesthesia (69\% [20 of 30]), followed by caudal (21\% [6 of 30]), thoracic epidural ( $10 \%$ [ 3 of 30]), and paravertebral or intercostal blocks (21\% [6 of 30]). Some respondents used more than 1 regional technique, hence the number of responses exceed the total number of respondents.

\section{Management During $C P B$}

The majority of the centers (40\% [36 of 91] used a target temperature during $\mathrm{CPB}$ that was tailored according to the complexity of the procedure. The remaining centers maintained normothermia (11\% [10 of 91]), allowed the temperature to drift passively (20\% [18 of 91]), or used target temperatures around $32^{\circ} \mathrm{C}$ to $34^{\circ} \mathrm{C}(23 \%$ [22 of 91]). A target temperature $<32^{\circ} \mathrm{C}$ was used routinely by only $5 \%$ ( 5 of 91 ) of the respondents.

Modified ultrafiltration (MUF) during CPB was used by $58 \%$ (40 of 69) of the respondents practicing OTE.

\section{Postoperative Management}

The majority of OTE patients (74\% [51 of 69]) were transferred to the PICU after OTE (other OTE patients were admitted to mixed adult and pediatric high-dependency units). The PICU unit was supervised by pediatricians (38\% [26 of 69]), cardiothoracic intensivists (22\% [15 of 69]), anesthesiologists (22\% [15 of 69]), general intensivists (1\% [1 of 69]), or others (17\% [12 of 69]). 


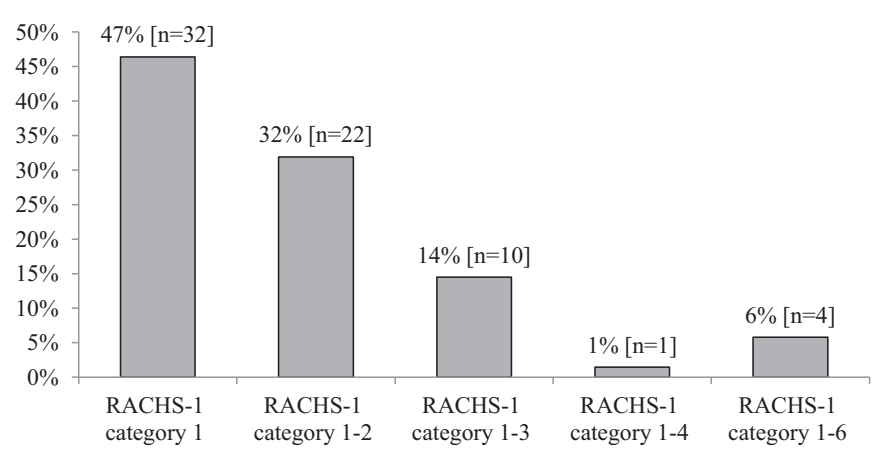

Fig. 4. Proportion of centers that perform on-table extubation in the various Risk Adjustment for Congenital Heart Surgery-1 categories. RACHS-1, Risk Adjustment for Congenital Heart Surgery-1.

The PICU unit staff was comfortable with the admission of extubated children from the OR according to $74 \%$ (51 of 69) of the OTE practitioners. Postoperative analgesia was maintained using multimodal analgesia techniques (opioid, nonsteroidal anti-inflammatory drugs, paracetamol, $\alpha_{2}$-receptor-agonists) by $74 \%$ (51 of 69) of the respondents, whereas the remaining pediatric cardiac anesthesiologists were using only a continuous infusion or intermittent boluses of opioids.

Fifty-one percent (41 of 69) of the respondents used noninvasive postoperative respiratory support if the respiratory situation was compromised. The main respiratory support strategies were continuous positive airway pressure, noninvasive ventilation, and high-flow oxygen through nasal cannula (Fig. 5). Respiratory physiotherapy was used by 51\% (27 of 53) of the respondents.

\section{Outcome and Safety}

Estimated median PICU length of stay for OTE patients varied from $<1$ to 1 to 2 days according to $34 \%$ (23 of 68) and $54 \%$ (37 of 68 ) of the respondents, respectively. In $12 \%$ (8 of 68 ) of the responding centers, patients stayed for 2 to 3 days in the ICU after OTE.

The reintubation rate was reported to be $<5 \%$ of OTE cases by $70 \%$ (48 of 69) of the respondents, whereas $25 \%$ (17 of 69) reported a reintubation rate of $5 \%$ to $10 \%$.

Safety was judged as being excellent by one-third of the respondents, whereas two-thirds considered their OTE practice still to be in an evolutionary stage. Three respondents abandoned OTE because practitioners in the unit to which OTE

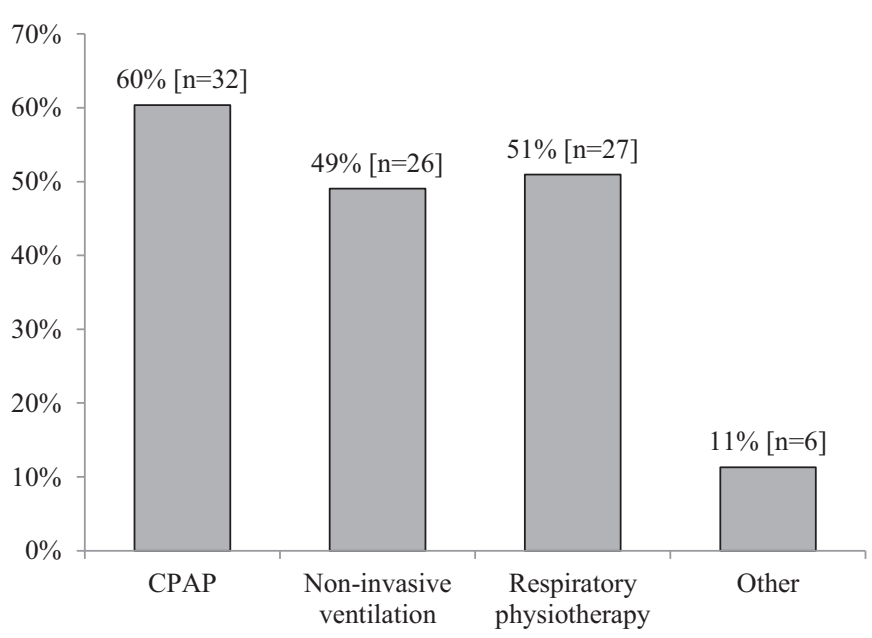

Fig. 5. Postoperative respiratory support strategies after on-table extubation (respondents could give more than 1 answer). CPAP, continuous positive airway pressure.

patients were transferred opposed the concept because they found OTE to be of no advantage.

\section{Reasons for not Practicing OTE}

OTE was not practiced by $24 \%$ (22 of 91 ) of the respondents. The main reasons given were that OTE was perceived to be of no major advantage (45\% [10 of 22]), that OTE decreased OR efficiency by increasing turnover times $(36 \%$ [8 of 22]), and that the cases were too complex to perform extubation on table (27\% [6 of 22]) (Fig. 6). Other reasons for not practicing OTE among the respondents were either the refusal by the cardiac surgeon or the PICU staff or that OTE was considered to be dangerous.

\section{Discussion}

To the best of the authors' knowledge, this is the first international survey aiming to describe the clinical practice of ultra-fast-track anesthesia with OTE among pediatric cardiac centers in Europe, North America, Asia, Oceania, and 2 African countries. The survey unveils multiple aspects regarding perceived feasibility, safety, perioperative anesthetic management, and postoperative care.

According to the results of the survey, OTE in pediatric cardiac surgery has become a clinical reality with OTE being practiced by the majority $(76 \%)$ of the respondents. However,

Table 1

Strategies Used for Intraoperative Analgesia

\begin{tabular}{|c|c|c|}
\hline Method & $\begin{array}{l}\text { Number of } \\
\text { Respondents }\end{array}$ & Percentage \\
\hline Low-dose opioid regimen ( $<20 \mu \mathrm{g} / \mathrm{kg}$ fentanyl or the equivalent dose of other opioids in total, with the exclusion of remifentanil) & 40 & $59 \%$ \\
\hline High-dose opioid regimen ( $>20 \mu \mathrm{g} / \mathrm{kg}$ fentanyl or the equivalent dose of other opioids in total, with the exclusion of remifentanil) & 5 & $7 \%$ \\
\hline Continuous infusion of remifentanil & 11 & $16 \%$ \\
\hline Opioid-free analgesia using only multimodal analgesia concepts & 2 & $3 \%$ \\
\hline Other & 10 & $15 \%$ \\
\hline
\end{tabular}




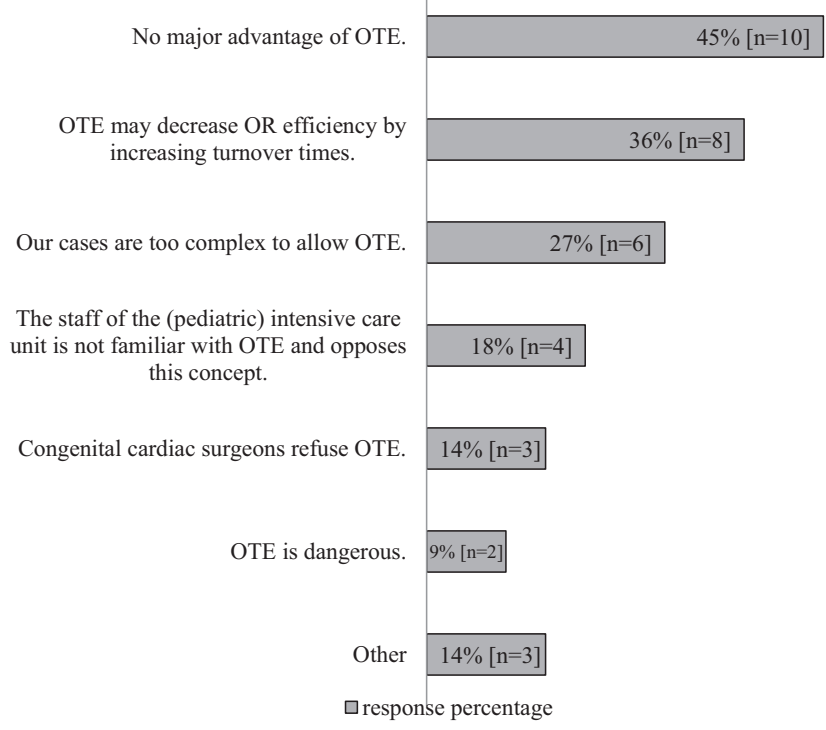

Fig. 6. Major reasons for not practicing on-table extubation (respondents could give more than 1 answer).

OTE still is far from being clinical routine in most centers, with approximately half of the respondents practicing OTE only occasionally. Interestingly, in 2 German centers, more than $51 \%$ of pediatric cardiac patients were extubated on table.

The majority of the practicing OTE centers used OTE either because of clinical considerations or to decrease the PICU turnover time. OTE has been associated with improved resource usage by shortening the ICU and hospital length of stays in a range of pediatric patients with $\mathrm{CHD} .^{10,11}$

It has been demonstrated that OTE is safer in cardiac cases of lower complexity, including those in the RACHS-1 categories 1 and $2 .{ }^{12-15}$ In an observational study with the objective to identify risk factors for extubation failure in the OR among pediatric cardiac surgical patients, $92 \%$ of patients (412 of 448) were extubated successfully in the OR with the success rate of OTE decreasing with increasing RACHS-1 categories: category $1(100 \%)$, category $2(90 \%)$, category $3(93 \%)$, and category $4(87 \%))^{7}$ Accordingly, the majority of respondents perform OTE in only low-risk categories. Notably, the authors identified 2 centers in Germany and 2 centers in The Netherlands that also practice OTE in the most complex procedures, including RACHS- 1 categories 4 to 6 .

Interestingly, in the majority of the centers, no formal criteria are followed for the inclusion of an individual patient in the OTE program. Instead, the decision mostly is made on a case-to-case basis by either the pediatric cardiac anesthesiologist alone or within the team. Only $5 \%$ of the responding pediatric cardiac anesthesiologists in the current survey followed formal inclusion or exclusion criteria for performing OTE.

When starting an OTE program, it is mandatory to identify patients for whom OTE might be contra-indicated. Exclusion criteria that are most frequently applied by OTE practitioners are evident and include various factors indicating actual or imminent instability, such as excessive bleeding, lower-thanexpected oxygen saturations for the type of surgical correction or palliation, long CPB times ( $>120$ minutes), and high inotropic or vasopressor support. The decision whether to perform OTE always should be based on a risk-to-benefit ratio for the individual patient that takes into account preoperative, intraoperative, and immediate postoperative factors.

Age was not considered to be an exclusion criterion by $55 \%$ of the respondents. This is supported by a recent study that demonstrated a weight $<4 \mathrm{~kg}$ but not age was associated with OTE failure. ${ }^{16}$ However, in that study, children with lower weight also underwent more complex procedures, which might represent an important confounder. In the survey described here, addressees were not specifically asked whether they consider weight as an exclusion criterion.

Application of appropriate intraoperative anesthesia protocols using short-acting opioids is vital when planning for OTE. ${ }^{17}$ In the present survey, dexmedetomidine was used by $31 \%$ of respondents as an adjunct to general anesthesia. This is in accordance with the findings of a recent analysis of the Congenital Cardiac Anesthesia Society-Society of Thoracic Surgeons registries, for which a growing use of dexmedetomidine was documented together with reduced mortality in children who receive this $\alpha_{2}$-receptor-agonist. ${ }^{18}$

Effective postoperative analgesia is a cornerstone for planning extubation in the $\mathrm{OR}^{7}$ Postoperative analgesia in the present survey was described as mainly being achieved with multimodal analgesia techniques in the majority of centers practicing OTE. More than half (57\%) of the pediatric cardiac anesthesiologists were not using regional anesthesia techniques for perioperative analgesia. Anesthesiologists in the remaining centers were using regional anesthesia techniques either infrequently $(31 \%)$ or routinely $(12 \%)$. Among the different regional anesthesia techniques, local infiltration analgesia was most commonly performed. Although several authors have described the successful use of neuraxial techniques, including thoracic epidural and caudal anesthesia, in pediatric cardiac surgery with high OTE rates, ${ }^{19,20}$ the safety and benefits these techniques still remain to be established. ${ }^{21,22}$

A MUF strategy during CPB was used by $60 \%$ of the pediatric cardiac anesthesiologists practicing OTE. MUF after separation from extracorporeal circulation has been reported to improve hemodynamics, pulmonary function, and coagulation parameters and to ameliorate organ dysfunction. Decrease in blood transfusion and reductions of total body water and postoperative blood loss are other advantages of MUF. ${ }^{23}$

Safety of OTE was perceived as being excellent, with an estimated ICU reintubation rate $<5 \%$ according to the majority $(70 \%)$ of the responding pediatric cardiac anesthesiologists. This reintubation rate is in accordance with the incidence of $2.4 \%$ recently reported in a large observational study. ${ }^{7}$ Importantly, none of the respondents had stopped OTE for safety reasons.

Of note, nearly one quarter of the survey respondents did not perform OTE, either for medical or logistical reasons. The majority of these centers were located in both European countries (United Kingdom and Turkey) and non-European countries (India and Bahrain); therefore, geographic influences seem to be of lesser importance for this reluctance. 


\section{Limitations}

The present survey had a relatively high response rate of $63 \%$, which is significantly greater than in other online surveys in the field of cardiac anesthesia, including $28 \%, 14 \%$, and $13 \%$ for surveys on perioperative fluid management, ${ }^{24}$ current use of the pulmonary artery catheter, ${ }^{25}$ and management of diastolic dysfunction, ${ }^{26}$ respectively.

Assessing practice is difficult because it usually varies, not only from institution to institution but also among physicians, even those in the same division. Several factors may influence the use of OTE, including surgical complexity, pathophysiology of the underlying condition, and physician preferences. The authors acknowledge that the respondents might represent an arbitrary selection of above-the-average interested pediatric cardiac anesthesiologists being engaged in a professional society or personally known to the authors. This makes the results prone to selection bias. Moreover, some countries were clearly underrepresented (eg, the United States, Canada, African countries). In other countries, however, respondents represented more than one-third (Germany) ${ }^{27}$ or nearly all pediatric cardiac centers (The Netherlands, Sweden). Nevertheless, given the variance in representativeness, a sampling error may have occurred that might have affected the generalizability of the results. Moreover, important questions regarding intraoperative hemodynamic and echocardiographic monitoring; conduct of CPB (type of circuits, priming, type of cardioplegia); and the preferred mode of ventilation were not addressed in an attempt to not overload the already comprehensive questionnaire. The survey also did not distinguish between on-pump and off-pump surgery. On the one hand, it could be argued that because of the avoidance of CPBinduced systemic inflammation, off-pump procedures are particularly appropriate for OTE. Moreover, several off-pump procedures are performed in children with a low perioperative risk (eg, aortic coarctectomies). Therefore, the inclusion of off-pump procedures in the survey might bias the results. On the other hand, a considerable proportion of off-pump procedures are performed in children with a high perioperative risk profile (eg, placement of a systemic-to-pulmonary shunt or pulmonary artery banding in a neonate with single ventricle physiology). The authors therefore could not justify excluding off-pump cardiac surgery from the survey.

Furthermore, important outcome parameters, including safety, effect on OR turnover times, extubation failure, length of stay, and cost effectiveness, could not be formally measured within the restrictions of a survey but had to be assessed as perceptions by the respondents. Moreover, the survey was unable to describe the actual extent of OTE practice because participants had to answer in estimated percentages rather than in exact numbers. For the accurate measurement of OTE prevalence, a prospective observational trial would be required.

\section{Conclusions}

Ultra-fast-track anesthesia with OTE is practiced regularly in pediatric cardiac surgery in the majority of the responding pediatric cardiac centers all over the world. Frequencies of
OTE and inclusion criteria vary widely. The observations made in this survey should prompt appropriately powered, randomized controlled clinical trials in which the effect of OTE on various effectiveness and safety outcomes should be tested.

\section{Questionnaire}

1 What kind of institution are you affiliated with?

- University hospital

- Children's hospital

- Heart center

- Maximum care hospital (neither university hospital nor heart center)

- Other (please specify)

2 In which country is your institute located?

3 How many patients $\leq 16$ years of age undergo cardiac surgery at your center every month?

- $<10$

- $10-20$

- 20-30

- $>30$

4 What is the proportion of on-pump congenital cardiac surgery in your pediatric population?

- $10 \%$

- $10 \%-50 \%$

- $>50 \%$

5 Complexity of cases being operated at your center:

- Operations of simple heart defects and vascular malformations

- Operations of all heart defects and vascular malformations including single ventricle pathology but excluding heart transplantation

- Operations of all heart defects and vascular malformations including single ventricle pathology and heart transplantation

6 What is the perceived time point of extubation in your pediatric intensive care unit (PICU) for children after cardiac surgery?

- In nearly all cases, within 6 hours after admission to the PICU

- The majority of uncomplicated cases are extubated within 6 hours after admission to the PICU

- Even in uncomplicated cases, more than 6 hours after admission to the PICU

- Children are usually extubated at the following morning earliest

- Other (please specify)

7 What is the target temperature (in degrees Celsius) that is used during cardiopulmonary bypass in the majority of pediatric cardiac procedures at your center?

- 36 (normothermia)

- 34-35 ("passive drift")

- $32-34$

- $<32$

- Depends on the complexity of the procedure

- Other (please specify) 
8 Have you ever practiced on-table extubation (OTE) in pediatric congenital cardiac surgery?

- Yes

- No

If "yes," the survey will continue through question 10

If "no," survey will end by answering question 9

9. What are the main reasons that have held you back from ever trying OTE or from performing it more frequently at your institution? You can give more than one answer.

- No major advantage of OTE

- OTE is dangerous

- The staff of the (pediatric) intensive care unit is not familiar with OTE and opposes this concept

- Congenital cardiac surgeons refuse OTE

- OTE may decrease operating room (OR) efficiency by increasing turnover times

- Our cases are too complex to allow OTE

- Other (please specify)

10 In what percentage of your cases do you perform on-table extubation (OTE) in congenital cardiac surgery?

- Very infrequently

- Regularly, in $1 \%-10 \%$ of the cases

- Regularly, in $11 \%-20 \%$ of the cases

- Regularly, in $21 \%-50 \%$ of the cases

- Regularly, in $>51 \%$ of the cases

- Other

11 Years since the practice of OTE has started at your center:

- $<5$ years

- 5 years

12 What are main reasons to perform OTE at your institution? You can give more than one answer.

- Logistical reasons (eg, scarcity of PICU resources)

- Increase in efficiency (increased intensive care unit [ICU] turnover by reducing ventilation times)

- Clinical considerations (eg, avoidance of positive pressure ventilation in patients with pulmonary hypertension or with Fontan circulation or restrictive right ventricular (RV) physiology like tetralogy of Fallot)

13 What are the exclusion criteria for OTE with respect to age in your institution?

- Age is not an exclusion criterion in our center

- $<6$ months

- $<12$ months

- $<6$ years

- $<12$ years

- Other (please specify)

14 When do you decide not to perform a planned OTE? You can give more than one answer.

- Excessive bleeding.

- Long CPB time (> 120 minutes)

- Percentage saturation of Oxygen $\left(\mathrm{SpO}_{2}\right)$ lower than expected for the type of surgical correction/palliation

- Dependency upon vasopressors/inotropes.

- All of the above.

- Other (please specify)
15 The decision to include/exclude a patient into OTE program is based on:

- Formal criteria

- Informal criteria (clinical experience, "gut feeling")

- The decision is made on an individual case-by-case basis

- Other (please specify)

16 In which RACHS-1 category cases do you perform OTE at your center?

- Only RACHS-1 category 1 (atrial septal defect repair, partial anomalous pulmonary venous connection surgery)

- RACHS-1 category 1-2 (see above, plus the following: ventricular septal defect (VSD) repair, tetralogy o fallout (TOF) total correction, Glenn shunt, repair of Aortopulmonary (AP) window)

- RACHS-1 category 1-3 (see above, plus the following: Fontan procedure, Right ventricle (RV) to Pulmonary artery (PA) conduit, arterial switch operation)

- RACHS-1 category 1-4 (see above, plus the following: arterial switch with VSD closure, unifocalization for TOF and pulmonary atresia, Konno procedure, truncus repair)

- RACHS-1 category 1-5 (see above, plus the following: tricuspid valve repositioning for neonatal Ebstein's anomaly ( $\leq 30$ days of age), repair of truncus arteriosus and interrupted arch)

- RACHS-1 category 1-6 (see above, plus the following: Norwood operation)

17 Who decides about OTE?

- Pediatric cardiac anesthesiologist

- Pediatric cardiac surgeon

- Integrated decision

- Other (please specify)

18 To which unit are children admitted after OTE?

- Postoperative anesthesia care unit

- Pediatric intensive care unit

- Mixed adult and pediatric ICU, specialized in cardiothoracic surgical patients

- Mixed general adult and pediatric ICU

- Other (please specify)

19 The unit to which children are admitted after cardiac surgery in your institution is led by:

- Pediatricians

- Cardiothoracic intensivists

- General intensivists

- Anesthesiologists

- Other (please specify)

20 Is the staff in the unit to which children is admitted after cardiac surgery in your institution comfortable with the admission of extubated children from the OR?

- Yes

- No

21 What is your (estimated) reintubation rate in the OTE cohort (within the first 6 hours after extubation)?

$-<5 \%$

$\bullet<10 \%$ 
- $>10 \%$

- Other (please specify)

22 How do you judge the current safety and feasibility of OTE at your center?

- Excellent

- OTE is still in evolution (we are in the process of finetuning our practices)

- $\mathrm{Bad}$ (we are considering to stop this program)

- We have stopped the practice of OTE

23 How do you maintain anesthesia in pediatric patients planned for OTE?

- With only volatile anesthetics

- Using only continuous intravenous anesthesia

- Using both volatile anesthetics and intravenous anesthesia

If option "first choice," question 25 will be skipped

24 Do the children receive postoperative nausea and vomiting (PONV) prophylaxis routinely intraoperatively?

- Yes

- No

25 Which intravenous anesthetics do you use for the maintenance of anesthesia (more than one option possible)?

- Propofol

- Benzodiazepines

- Ketamine

- Dexmedetomidine

- Clonidine

- Barbiturates

- Other (please specify)

26 Do the children receive PONV prophylaxis routinely intraoperatively?

- Yes

- No

27 How do you perform analgesia in congenital cardiac surgery planned for OTE?

- Low dose opioid regimen (less than $20 \mu \mathrm{g} / \mathrm{kg}$ fentanyl or the equivalent dose of other opioids in total, with the exclusion of remifentanil)

- High dose opioid regimen (more than $20 \mu \mathrm{g} / \mathrm{kg}$ fentanyl or the equivalent dose of other opioids in total, with the exclusion of remifentanil)

- Continuous infusion of remifentanil

- Opioid-free analgesia using only multimodal analgesia concepts

- Other (please specify)

28 Do you use regional anesthesia in OTE patients?

- Never

- Yes

- Sometimes

If "never," question 29 will be skipped

29 What kind of regional anesthesia techniques do you use in OTE patients (more than one option possible)?

- Caudal anesthesia

- Thoracic epidural anesthesia
- Local infiltration anesthesia

- Other (please specify)

30 How is postoperative analgesia achieved after OTE (more than one option possible)?

- Continuous infusion of opioids

- Intermittent administration of opioids

- Intermittent administration of nonsteroidal anti-inflammatory drugs

- Intermittent administration of acetaminophen

- Loco-regional anesthesia

- Multimodal analgesia (opioids, nonsteroidal anti-inflammatory drug, paracetamol)

- Regional anesthesia

- Other (please specify)

31 Do you routinely use modified ultrafiltration (MUF) in OTE patients?

- Yes

- No

32 Do children with OTE prophylactically receive respiratory support in your institution (post-OTE in PICU)?

- Yes

- No

- Only when the respiratory situation is compromised

If "no," question 33 will be skipped

33 What is the preferred technique of postoperative respiratory support in your institution for children after OTE (more than one option possible)?

- Continuous positive airway pressure

- Noninvasive ventilation

- Respiratory physiotherapy

- Other (please specify)

34 What is the median length of stay in the PICU for patients having OTE?

- $<1$ day

- 1-2 days

- 2-3 days

- 3 days

\section{References}

1 Wong WT, Lai VKW, Chee YE, et al. Fast-track cardiac care for adult cardiac surgical patients. Cochrane Database Syst Rev 2016;12:9.

2 Ender J1, Borger MA, Scholz M, et al. Cardiac surgery fast-track treatment in a post-anesthetic care unit: Six-month results of the Leipzig fast-track concept.. Anesthesiology 2008;109:61-6.

3 Montes FR, Sanchez SI, Giraldo JC, et al. Lack of benefit of tracheal extubation in operating room after CABG. Anaesth Analg 2009;91:776-80.

4 Hemmerling TM, Lê N, Olivier JF, et al. Immediate extubation after aortic valve surgery using high thoracic epidural analgesia or opioid-based analgesia. J Cardiothorac Vasc Anesth 2005;19:176-81.

5 Salah M, Hosny H, Salah M, et al. Impact of immediate versus delayed tracheal extubation on length of ICU stay of cardiac surgical patients, a randomized trial. Heart Lung Vessel 2015;7:311-9.

6 Makhija Z, Kumar A, Bhatt M. Effect of on-table extubation after congenital heart surgery on outcomes in a developing country. J Cardiothoracic Surg 2015;10:281 
7 Joshi RK, Aggarwal N, Agarwal M, et al. Assessment of risk factors for a sustainable "on-table extubation" program in pediatric congenital cardiac surgery: 5-year experience. J Cardiothorac Vasc Anesth 2016;30:1530-8.

8 Harris KC, Holowachuk S, Pitfield S, et al. Should early extubation be the goal for children after congenital cardiac surgery? J Thorac Cardiovasc Surg 2014;148:2642-8.

9 Abuchaim DC, Bervanger S, Medeiros SA, et al. Early extubation of children in the operating room after cardiac surgery. Rev Bras Cir Cardiovasc 2010;25:103-8.

10 Lawrence EJ, Nguyen K, Morris SA, et al. Economic and safety implications of introducing fast tracking in congenital heart surgery. Circ Cardiovasc Qual Outcomes 2013;6:201-7.

11 Howard F, Brown KL, Garside V, et al. Fast-track paediatric cardiac surgery: The feasibility and benefits of a protocol for uncomplicated cases Eur J Cardiothorac Surg 2010;37:193-6.

12 Hoda M, Haque A, Aijaz F, et al. On-table extubation after open heart surgery in children: An experience from a tertiary care hospital in a developing country. Congenit Heart Dis 2016;11:58-62.

13 Vricella LA, Dearani JA, Gundry SR, et al. Ultra-fast track in elective congenital cardiac surgery. Ann Thorac Surg 2000;69:861-5.

14 Akhtar MI, Hamid M, Anwar-Ul-Haq, et al., et al. Feasibility and safety of on table extubation after corrective surgical repair of tetralogy of Fallot in a developing country. A case series. Ann Card Anaesth 2015;18:237.

15 Akhtar MI, Hamid M, Minai F, et al. Safety profile of fast track extubation in pediatric congenital heart disease (CHD) surgery patients in a tertiary care hospital of a developing country. An observational prospective study. J Anaesthesiol Clin Pharmacol 2014;30:355-9.

16 Miller JW, Vu Dien, Chai PJ. Patient and procedural characteristics for successful and failed immediate tracheal extubation in the operating room following cardiac surgery in infancy. Paediatr Anaesth 2014;24:830-9.
17 Iezzi F, di Summa M, Del Sarto P, et al. Fast-track extubation in pediatric cardiothoracic surgery in developing countries. J Card Crit Care TSS 2017;1:18-20.

18 Schwartz LI, Twite M, Gulack B, et al. The perioperative use of dexmedetomidine in pediatric patients with congenital heart disease: An analysis from the Congenital Cardiac Anesthesia Society-Society of Thoracic Surgeons Congenital Heart Disease database. Anesth Analg 2016;123:715-21.

19 Peterson KL, DeCampli WM, Pike NA, et al. A report of 220 consecutive cases of regional anesthesia in pediatric cardiac surgery. Anesth Analg 2000;90:1014-9.

20 Mittnacht AJ, Thanjan M, Srivastava S, et al. Extubation in the operating room after congenital heart surgery in children. J Thorac Cardiovasc Surg 2008; 136:88-93.

21 Rosen DA, Hawkinberry DW, Rosen KR, et al. An epidural hematoma in an adolescent patient after cardiac surgery. Anesth Analg 2004;98:966-9.

22 Chaney MA. Thoracic epidural anesthesia in cardiac surgery - the current standing. Ann Card Anaesth 2009;12:1-3.

23 Ziyaeifard M, Alizadehasl A, Massoumi G. Modified ultrafiltration during cardiopulmonary bypass and postoperative course of pediatric cardiac surgery. Res Cardiovasc Med 2014;3:e17830.

24 Protsyk V, Rasmussen BS, Guarracino F, et al. Fluid management in cardiac surgery: Results of a survey in European cardiac anesthesia departments. J Cardiothorac Vasc Anesth 2017;31:1624-9.

25 Judge $\mathrm{O}$, Ji F, Fleming N, et al. Current use of the pulmonary artery catheter in cardiac surgery: A survey study. J Cardiothorac Vasc Anesth 2015;29:69-75.

26 McIlroy DR, Lin E, Hastings S, et al. Intraoperative transesophageal echocardiography for the evaluation and management of diastolic dysfunction in patients undergoing cardiac surgery: A survey of current practice. J Cardiothorac Vasc Anesth 2016;30:389-97.

27 Baehner T, Dewald O, Heinze I, et al. The provision of pediatric cardiac anesthesia services in Germany: Current status of structural and personnel organization. Paediatric Anaesth 2017;27:801-9. 